\title{
Towards flavour diffusion coefficient and electrical conductivity without ultraviolet contamination
}

\author{
Y. Burnier ${ }^{1}$ and M. Laine ${ }^{2}$ \\ 1 Institute for Theoretical Physics, Albert Einstein Center, University of Bern, Sidlerstrasse 5, CH-3012 Bern, Switzerland \\ 2 Faculty of Physics, University of Bielefeld, D-33501 Bielefeld, Germany
}

February 2012

BI-TP 2012/01

\begin{abstract}
By subtracting from a recent lattice measurement of the thermal vector-current correlator the known 5-loop vacuum contribution, we demonstrate that the remainder is small and shows no visible shortdistance divergence. It can therefore in principle be subjected to model-independent analytic continuation. Testing a particular implementation, we obtain estimates for the flavour-diffusion coefficient $(2 \pi T D \gtrsim 0.8)$ and electrical conductivity which are significantly smaller than previous results. Although systematic errors remain beyond control at present, some aspects of our approach could be of a wider applicability.
\end{abstract}

\section{Introduction}

If a plasma is subjected to a perturbation whose wavelength is much longer than the typical mean-free path, then decohering scatterings take abundantly place within that perturbation, and its macroscopic physics should be classical in nature. For instance, if we imagine a hadronic "jet" with a width of a fermi or more, placed within a strongly interacting medium at a temperature $T \gg 200 \mathrm{MeV}$, in which the characteristic distance scale is $1 /(\pi T) \ll \mathrm{fm}$, then we expect to be able to describe the gross features of the jet's subsequent behaviour within classical hydrodynamics. The main role of quantum physics is that the classical description involves parameters, called transport coefficients, which need to be matched to the fundamental theory, in order to correctly capture the dynamics.

As a particular case, let us consider a perturbation carrying a specific net quark flavour. In the absence of weak interactions, there is a conserved current related to each flavour: $\partial_{\mu} J_{f}^{\mu}=0, f=1, \ldots, N_{\mathrm{f}}$. The sum of all flavour currents (divided by the number of colours, $N_{\mathrm{c}}$ ) defines the baryon current, whereas a particular linear combination, weighted by the electric charges of all flavours, defines the electromagnetic current (denoted by $J_{\mathrm{em}}^{\mu}$ ).

Now, to define the flavour diffusion coefficient, denoted by $D_{f}$, requires a specification of the classical description onto which to match. Let us assume that the perturbation is broad compared with $1 /(\pi T)$, and express $J_{f}^{\mu}$ in a gradient expansion in $\sim \partial^{\mu} / \pi T$. Apart from $\partial^{\mu}$ the other Lorentz vector available is the four-velocity defining the fluid rest frame $\left(u^{\mu} ; u^{\mu} u_{\mu}=1\right)$. The free coefficients allowed by Lorentz symmetry are the transport coefficients. In particular, we can expand

$$
J_{f}^{\mu}=u^{\mu} n_{f}+D_{f} \partial_{\perp}^{\mu} n_{f}+\mathcal{O}\left(\partial^{2}\right) \quad(f \text { fixed }),
$$

where $n_{f}$ is the number density, and the transverse derivative has been defined as

$$
\partial_{\perp}^{\mu} \equiv\left(\eta^{\mu \nu}-u^{\mu} u^{\nu}\right) \partial_{\nu}, \quad \eta^{\mu \nu} \equiv \operatorname{diag}(+---) .
$$

The so-called Landau-Lifshitz convention is implied here, whereby in the rest frame of the fluid the zero component of $J_{f}^{\mu}$ is nothing but the number density: $u_{\mu} J_{f}^{\mu} \equiv n_{f}$.

To match for $D_{f}$, it is helpful to specialize to simple kinematics. In particular, going to the fluid rest frame and imposing current conservation on eq. (1.1) yields

$$
\partial_{t} n_{f}=D_{f} \nabla^{2} n_{f}+\mathcal{O}\left(\nabla^{3}\right) .
$$

In this frame it is a textbook exercise to derive an expression for $D_{f}$ in terms of 2-point correlators, which can then be matched onto quantum-mechanical expectation values; the result reads (cf. e.g. ref. [1])

$$
D_{f}=\frac{1}{3 \chi_{f}} \lim _{\omega \rightarrow 0} \sum_{i=1}^{3} \frac{\rho_{i i}(\omega, \mathbf{0})}{\omega}
$$

where $\rho_{i i}(\omega, \mathbf{0}) \equiv \int_{t, \mathbf{x}} e^{i \omega t}\left\langle\frac{1}{2}\left[\hat{J}_{f}^{i}(t, \mathbf{x}), \hat{J}_{f}^{i}(0, \mathbf{0})\right]\right\rangle_{T}$ is the spectral function related to the operators $\hat{J}_{f}^{i}$, and

$$
\begin{aligned}
\chi_{f} & \equiv \int_{0}^{\beta} \mathrm{d} \tau \int_{\mathbf{x}}\left\langle\hat{J}_{f}^{0}(\tau, \mathbf{x}) \hat{J}_{f}^{0}(0, \mathbf{0})\right\rangle_{T} \\
& =\beta \int_{\mathbf{x}}\left\langle\hat{J}_{f}^{0}(0, \mathbf{x}) \hat{J}_{f}^{0}(0, \mathbf{0})\right\rangle_{T}, \quad \beta \equiv \frac{1}{T},
\end{aligned}
$$

is the "susceptibility" related to the conserved charge. It is important to stress that even though this way of determining $D_{f}$ makes use of the fluid rest frame (reflected by the vanishing spatial momentum in eq. (1.4); in the following this redundant argument is suppressed), the coefficient 
itself is defined also for relativistic flow; the corresponding covariant form of the diffusion equation is eq. (1.1) together with $\partial_{\mu} J_{f}^{\mu}=0$.

In the case of the electrical conductivity, $\sigma$, a possible definition is $\left\langle\hat{\mathbf{J}}_{\mathrm{em}}\right\rangle=\sigma \mathbf{E}$, where $\mathbf{E}$ denotes an external electric field. Let us denote $D_{f}=D_{f, \mathrm{~s}}+D_{f, \mathrm{~ns}}$, $\chi_{f}=\chi_{f, \mathrm{~s}}+\chi_{f, \mathrm{~ns}}$, where $D_{f, \mathrm{~s}}, \chi_{f, \mathrm{~s}}$ are the contributions of the "singlet" or "disconnected" quark contractions, and $D_{f, \mathrm{~ns}}, \chi_{f, \mathrm{~ns}}$ those of the "non-singlet" or "connected" ones. One then obtains

$$
\sigma=e^{2} \chi_{f}\left[\left(\sum_{f=1}^{N_{\mathrm{f}}} Q_{f}\right)^{2} D_{f, \mathrm{~s}}+\left(\sum_{f=1}^{N_{\mathrm{f}}} Q_{f}^{2}\right) D_{f, \mathrm{~ns}}\right]
$$

where $Q_{f}$ denotes the electric charge of flavour $f$ in units of the elementary charge $e$.

Although a lattice Monte Carlo determination of $D_{f}$ and $\sigma$ is numerically very demanding [2], a number of attempts have been launched in recent years [3-5]. In particular, in ref. [5] a continuum extrapolation of the relevant (connected) Euclidean correlator was carried out for the first time, with a philosophy that analytic continuation from the Euclidean correlator to the Minkowskian spectral function should only be attempted on the continuumextrapolated result. Subsequently various models were employed for the analytic continuation, based on a fewparameter ansatz for $\rho_{i i}$. In the present work we make use of the continuum-extrapolated Euclidean data of ref. [5], but analyze it in a different way, avoiding fits.

The basic philosophy of our approach comes from ref. [6], whose results were transcribed into a practical algorithm in ref. [7]. The main point is that in order to allow for an analytic continuation in principle, short-distance divergences need to be subtracted from the Euclidean correlator, such that Fourier coefficients can be determined (in fact the function should even be continuous [6]). An important further insight comes from ref. [8], which showed that the ultraviolet (UV) asymptotics of the thermal contribution to the spectral function $\rho_{i i}$ is such that it does lead to a continuous Euclidean correlator. Therefore, only the contribution of the vacuum $\rho_{i i}$ needs to be subtracted. The final ingredient is that the vacuum $\rho_{i i}$ can be extracted from a recent 5-loop computation of the vector current correlator [9]. Implementing all these ingredients, we find surprisingly stable results which can be compared with other approaches, in order to obtain a rough impression on the systematic uncertainties involved.

\section{Detailed setup}

We have in mind QCD with three massless valence flavours $\left(N_{\mathrm{v}} \equiv 3\right)$. The gauge field configurations were generated within pure $\mathrm{SU}(3)$ gauge theory in ref. [5], so the number of dynamical quarks is zero $\left(N_{\mathrm{f}}=0\right)$. Moreover in ref. [5] only the "connected" or "non-singlet" contractions were evaluated. For $N_{\mathrm{v}}=3$ this implies that we are technically considering electric charge diffusion and susceptibility; following ref. [5] the corresponding coefficients are denoted by $D \equiv D_{f, \mathrm{~ns}}$ and $\chi_{\mathrm{q}} \equiv \chi_{f, \mathrm{~ns}}$. Otherwise we keep the notation of sec. 1 in the following, in particular continuing to employ Minkowskian conventions for the Dirac matrices. (Note that the physics of heavy flavour diffusion, referring to quarks with a mass $M \gg \pi T$, is quite different from that of light quark diffusion $[10,11]$, and the two cases should not be confused with each other, even though a single notation $D$ is often used for the diffusion coefficient; in particular, in the heavy quark case the extraction of $D$ might be somewhat more robust than here $[12,13]$.)

To specify the observables, it is helpful to start with the Lorentz covariant form of the vector current correlator,

$$
\begin{aligned}
G_{V}(\tau) & \equiv-\sum_{\mu=0}^{3} \int_{\mathbf{x}}\left\langle\left(\bar{\psi} \gamma_{\mu} \psi\right)(\tau, \mathbf{x})\left(\bar{\psi} \gamma^{\mu} \psi\right)(0, \mathbf{0})\right\rangle_{T} \\
& \equiv-G_{00}(\tau)+G_{i i}(\tau)
\end{aligned}
$$

where in the spatial part a sum over the indices is implied. Because of the projection to zero spatial momentum, $G_{00}(\tau)$ is actually $\tau$-independent, like in eq. (1.5); the value is denoted by $G_{00}(\tau)=\chi_{\mathrm{q}} T$. It turns out that in the free limit, the spatial $G_{i i}(\tau)$ contains the same constant, which then cancels in the sum of eq. (2.1) [14]. We denote this by $\chi_{\mathrm{q}}^{\text {free }}=N_{\mathrm{c}} T^{2} / 3[15]$. In the interacting theory, $G_{00}$ remains constant whereas $G_{i i}$ gets essentially modified.

The spectral functions corresponding to $G_{V}(\tau)$ and $G_{i i}(\tau)$ are denoted by $\rho_{V}(\omega)$ and $\rho_{i i}(\omega)$, respectively. If the spectral function is known, the Euclidean correlator can be obtained from

$$
G_{i i}(\tau)=\int_{0}^{\infty} \frac{\mathrm{d} \omega}{\pi} \rho_{i i}(\omega) \frac{\cosh \left(\frac{\beta}{2}-\tau\right) \omega}{\sinh \frac{\beta \omega}{2}}
$$

The basic issue is to what extent the inverse is true, i.e. information about the spectral function, particularly concerning the transport coefficient $\lim _{\omega \rightarrow 0^{+}} \rho_{i i}(\omega) / \omega$ relevant for eq. (1.4), can be extracted from a measured $G_{i i}$. An extensive review on the problems encountered and the methods currently available can be found in ref. [2].

We note in passing that the relation in eq. (2.1) implies a corresponding relation of the spectral functions, $\rho_{V}(\omega)=\rho_{i i}(\omega)-\pi \chi_{\mathrm{q}} \omega \delta(\omega)$. However the transport coefficients extracted from $\rho_{V}$ and $\rho_{i i}$ as $\lim _{\omega \rightarrow 0^{+}} \rho(\omega) / \omega$ are identical. In fact in the rigorous algorithm of ref. [6] the $\tau$-independent mode gets explicitly projected out.

Now, in ref. [8], the UV asymptotics of thermal spectral functions were analyzed with Operator Product Expansion methods. In particular, it was shown that thermal corrections to $\rho_{i i}(\omega)$ decrease at large frequencies as $\sim T^{4} / \omega^{2}$. When taken together with eq. (2.2) this statement, valid beyond perturbation theory, implies that the thermal part of $\rho_{i i}(\omega)$ yields a contribution to $G_{i i}(\tau)$ which is integrable even at $\tau=0$, i.e. remains finite at short distances. In contrast, the vacuum spectral function yields a contribution diverging as $\sim 1 / \tau^{3}$. In order for the rigorous analytic continuation of ref. [6] to be applicable, the divergent part needs to be subtracted [7]. 
In the literature, different strategies have been pursued for the subtraction. In particular, in refs. [16,17], analogous (but different) correlators were considered, and the idea was to compute the whole $G(\tau)$, including thermal corrections, up to next-to-leading order (NLO), i.e. 2-loop level, or $\mathcal{O}\left(\alpha_{s}\right)$. Although the same strategy would also be possible for the vector correlator, by making use of classic results for the NLO thermal spectral function [18-20], our goal here is to probe a different strategy. Namely, the vacuum subtraction is handled with much higher precision than NLO, by making use of the fact that in vacuum, $\rho_{i i}(\omega)$ is known up to 5-loop level, or $\mathcal{O}\left(\alpha_{s}^{4}\right)$ [9]. In contrast, the thermal part is handled with lower precision, only at leading order, leaving all other thermal effects to be taken care of by the non-perturbative numerical treatment of the remainder.

Before proceeding it is important to underline once more the implications of the asymptotic behaviour $\sim$ $T^{4} / \omega^{2}$ [8]. In particular, the Lorentzian form

$$
\rho_{i i}^{(\mathrm{L})}(\omega) \equiv 3 D \chi_{\mathrm{q}} \frac{\omega \eta^{2}}{\omega^{2}+\eta^{2}}
$$

is sometimes used for modelling the transport peak. However, this shape can be correct only at small frequencies [21]; at large frequencies $\omega \gg \eta$ it decays as $\sim$ $3 D \chi_{\mathrm{q}} \eta^{2} / \omega$, which is slower than the mentioned $\sim T^{4} / \omega^{2}$ (and does not allow for a negative sign which is also a possibility [8]). Most significantly, the Euclidean correlator obtained by inserting $\rho_{i i}^{(\mathrm{L})}$ into eq. (2.2) diverges at $\tau \ll \beta$. So, if used as a part of a fit ansatz for all $\omega$, this function may pick up an incorrect overlap on vacuum contributions, which could lead to an overestimate of $D$.

\section{Vacuum spectral function}

We now turn to ref. [9] and specify the 5-loop vacuum spectral function. Following the conventions of ref. [22], the coefficients of the $\beta$-function are defined according to

$$
\partial_{t} a_{s}=-\left(\beta_{0} a_{s}^{2}+\beta_{1} a_{s}^{3}+\beta_{2} a_{s}^{4}+\ldots\right),
$$

where

$$
a_{s} \equiv \frac{\alpha_{s}(\bar{\mu})}{\pi}, \quad t \equiv \ln \left(\frac{\bar{\mu}^{2}}{\Lambda_{\overline{\mathrm{MS}}}^{2}}\right),
$$

and, for $N_{\mathrm{c}}=3$ (cf. ref. [23]; we only need terms up to the 3-loop level here),

$$
\begin{aligned}
& \beta_{0}=\frac{11}{4}-\frac{N_{\mathrm{f}}}{6}, \\
& \beta_{1}=\frac{51}{8}-\frac{19 N_{\mathrm{f}}}{24}, \\
& \beta_{2}=\frac{2857}{128}-\frac{5033 N_{\mathrm{f}}}{1152}+\frac{325 N_{\mathrm{f}}^{2}}{3456} .
\end{aligned}
$$

The scale parameter $\Lambda_{\overline{\mathrm{MS}}}$ in eq. (3.2) represents an integration constant and, as usual, is chosen so that the asymptotic $(t \gg 1)$ behaviour reads

$a_{s}=\frac{1}{\beta_{0} t}-\frac{\beta_{1} \ln t}{\beta_{0}^{3} t^{2}}+\frac{\beta_{1}^{2}\left(\ln ^{2} t-\ln t-1\right)+\beta_{2} \beta_{0}}{\beta_{0}^{5} t^{3}}+\mathcal{O}\left(\frac{1}{t^{4}}\right)$.

Then, the main result can be obtained from the coefficients $\Pi_{i}^{0}, i=0, \ldots, 4$, in eqs. (6)-(9) of ref. [22]. In vacuum, the quantity that we are interested in can be written as

$$
\rho_{V}(\omega)=\frac{3 \omega^{2}}{4 \pi} R\left(\omega^{2}\right),
$$

where

$$
R\left(\omega^{2}\right)=4 \pi \operatorname{Im}\left\{\Pi_{0}^{0}+\Pi_{1}^{0} a_{s}+\Pi_{2}^{0} a_{s}^{2}+\ldots\right\}_{L \rightarrow \ell+i \pi},
$$

and

$$
\ell \equiv \ln \left(\frac{\bar{\mu}^{2}}{\omega^{2}}\right)
$$

Writing

$$
\begin{aligned}
R\left(\omega^{2}\right) & =r_{0,0}+r_{1,0} a_{s}+\left(r_{2,0}+r_{2,1} \ell\right) a_{s}^{2} \\
& +\left(r_{3,0}+r_{3,1} \ell+r_{3,2} \ell^{2}\right) a_{s}^{3} \\
& +\left(r_{4,0}+r_{4,1} \ell+r_{4,2} \ell^{2}+r_{4,3} \ell^{3}\right) a_{s}^{4}+\mathcal{O}\left(a_{s}^{5}\right)
\end{aligned}
$$

the generalization of eq. (5) of ref. [9] to $\ell \neq 0$ reads

$$
\begin{array}{r}
r_{0,0}=1.0000000, \\
r_{1,0}=1.0000000, \\
r_{2,0}=1.9857074-0.1152954 N_{\mathrm{f}}, \\
r_{2,1}=2.7500000-0.1666667 N_{\mathrm{f}}, \\
r_{3,0}=-6.6369356-1.2001341 N_{\mathrm{f}}-0.00517836 N_{\mathrm{f}}^{2}, \\
r_{3,1}=17.296391-2.0876938 N_{\mathrm{f}}+0.03843180 N_{\mathrm{f}}^{2}, \\
r_{3,2}=7.5625000-0.9166667 N_{\mathrm{f}}+0.02777778 N_{\mathrm{f}}^{2}, \\
r_{4,0}=-156.60811+18.774765 N_{\mathrm{f}}-0.79743434 N_{\mathrm{f}}^{2} \\
+0.021516105 N_{\mathrm{f}}^{3}, \\
r_{4,1}=-7.1166367-15.565615 N_{\mathrm{f}}+0.83393599 N_{\mathrm{f}}^{2} \\
+0.002589178 N_{\mathrm{f}}^{3}, \\
r_{4,2}=88.878862-16.175418 N_{\mathrm{f}}+0.81239907 N_{\mathrm{f}}^{2} \\
-0.009607950 N_{\mathrm{f}}^{3}, \\
r_{4,3}=20.796875-3.7812500 N_{\mathrm{f}}+0.22916667 N_{\mathrm{f}}^{2} \\
-0.004629630 N_{\mathrm{f}}^{3} .
\end{array}
$$

As already mentioned, the lattice simulations of ref. [5] were for quenched QCD $\left(N_{\mathrm{f}}=0\right)$ and only evaluated the "connected" quark contraction. In the language of refs. [9, 22 ] the latter corresponds to "non-singlet" contributions, which are the only ones included in the results above. Therefore, eqs. (3.10), (3.11) with $N_{\mathrm{f}}=0$ can directly be used for the analysis of the lattice data of ref. [5]. (Ultimately singlet contributions will need to be included as well, and on the perturbative side progress in this direction is being made, cf. e.g. ref. [24].) 


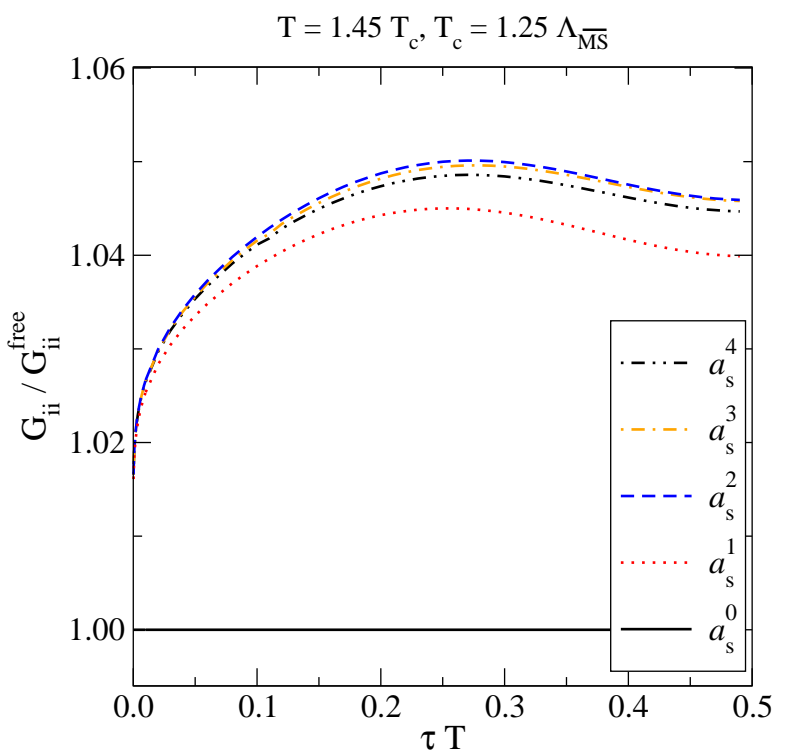

Fig. 1. Five orders of $G_{i i}(\tau)$, from eqs. (2.2), (3.10), (4.2), normalized to the free result from eq. (4.3). (The gauge coupling has always been evaluated with 3 -loop running.)

\section{Imaginary-time correlator}

Given the spectral function from eq. (3.7), re-interpreted as $\rho_{i i}$, we could insert it into eq. (2.2) and compute the corresponding imaginary-time correlator. There is the problem, though, that technically arbitrarily small values of $\omega$ contribute to the integral, but for those the determination of $a_{s}$ becomes ill-defined. However, any modification of $\rho_{i i}$ in a finite range $0 \leq \omega \leq \omega_{\max }$ yields a contribution to $G_{i i}(\tau)$ which does not diverge at small $\tau$ and can thus be taken care of together with the rest of the remainder. So, we cut the smallest frequencies off from $a_{s}$ by defining

$$
\bar{\mu}_{\text {ref }} \equiv \max (\pi T, \omega)
$$

and by choosing $\bar{\mu}=\bar{\mu}_{\text {ref }}$ in the evaluation of $G_{i i}(\tau)$. In addition we modify eq. (3.7) by accounting for the leadingorder thermal corrections:

$$
\rho_{i i}^{(T)}(\omega) \equiv \frac{3 \omega^{2}}{4 \pi}\left[1-2 n_{\mathrm{F}}\left(\frac{\omega}{2}\right)\right] R\left(\omega^{2}\right)+\pi \chi_{\mathrm{q}}^{\text {free }} \omega \delta(\omega),
$$

where $n_{\mathrm{F}}$ is the Fermi distribution. Following ref. [5] the temperature is set to $T=1.45 T_{\mathrm{c}}$, and following e.g. ref. [25] we take $T_{\mathrm{c}}=1.25 \Lambda_{\overline{\mathrm{MS}}}$ (uncertainties related to this choice are discussed below). Like in ref. [5] the results are normalized to the expression [26]

$$
\begin{gathered}
G_{i i}^{\mathrm{free}}(\tau) \equiv \int_{-\infty}^{\infty} \frac{\mathrm{d} \omega}{2 \pi}\left\{\frac{3 \omega^{2}}{4 \pi}\left[1-2 n_{\mathrm{F}}\left(\frac{\omega}{2}\right)\right]+\pi T^{2} \omega \delta(\omega)\right\} \\
\times \frac{\cosh \left(\frac{\beta}{2}-\tau\right) \omega}{\sinh \frac{\beta \omega}{2}} \\
=6 T^{3}\left[\pi(1-2 \tau T) \frac{1+\cos ^{2}(2 \pi \tau T)}{\sin ^{3}(2 \pi \tau T)}\right. \\
\left.+\frac{2 \cos (2 \pi \tau T)}{\sin ^{2}(2 \pi \tau T)}+\frac{1}{6}\right]
\end{gathered}
$$

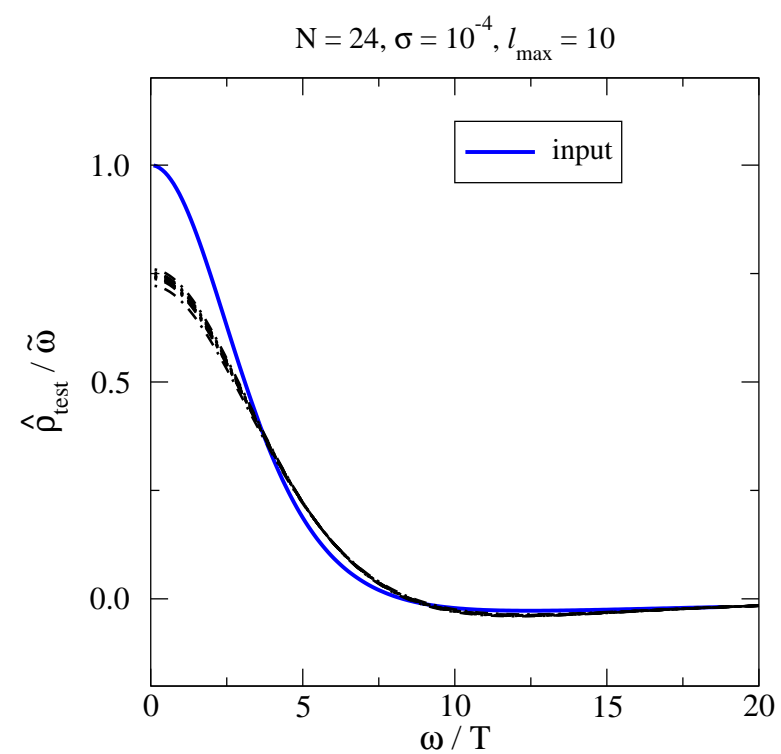

Fig. 3. A test of the method of ref. [6] with the example introduced in ref. [7], employing an improved prescription. Here $\hat{\rho}$ refers to normalization by appropriate powers of $T$; and "input" denotes the correct result, known in this case. The result should be compared with fig. 4(right) of ref. [7], where an identical data set lead to considerably more spread.

In fig. 1, five subsequent orders of the perturbative result are shown; in fig. 2 the 5-loop perturbative result is compared with the continuum-extrapolated data from ref. [5].

\section{Analytic continuation}

In order to estimate $D$ from eq. (1.4), data for the Euclidean $G_{i i}(\tau)$ should be fed into an analytic continuation prescription, yielding the corresponding $\rho_{i i}(\omega)$. The function $G_{i i}^{\text {pert }}$ based on eq. (4.2) yields a vanishing $D$, because $\rho_{i i}^{(T)}$ has a vanishing slope around the origin. Therefore we can equally well apply analytic continuation to the difference $G_{i i}^{\text {latt }}-G_{i i}^{\text {pert }}$. In fact, not only are we allowed to do this, but we probably must do this, given that to our knowledge mathematically justified analytic continuation to Minkowskian signature has been worked out only for a function which has no singularity at small $\tau[6]$.

After the subtraction of the singular terms, we make use of the method of ref. [6], as implemented in ref. [7]. In the meanwhile we have even found a significant improvement over the original implementation. The general method is based on determining the Fourier coefficients from the Euclidean data $G(\tau)$, which can be done numerically, and using these in order to construct an expansion of the real-time dependence of the correlator in terms of Laguerre polynomials, with coefficients denoted by $a_{\ell}$. The physical real-time correlator must vanish at infinite time separation [27], which corresponds to $\sum_{\ell=0}^{\ell_{\max }} a_{\ell}=0$. In practice, however, the correlator does not vanish exactly; choices of $\ell_{\max }$ for which it vanishes approximately were dubbed "windows of opportunity" in ref. [7]. Within 

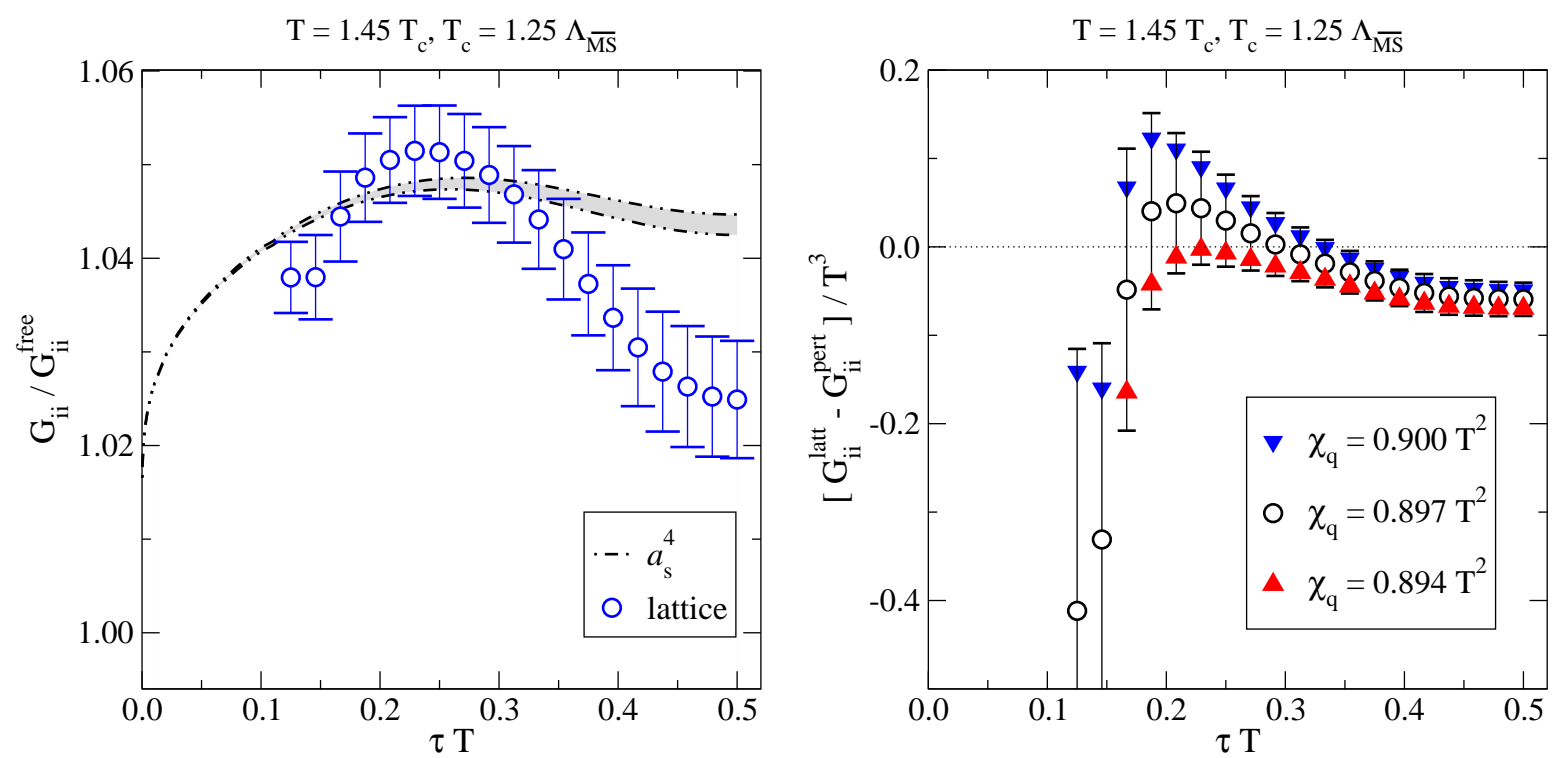

Fig. 2. Left: Comparison of the $\mathcal{O}\left(a_{s}^{4}\right)$ result from fig. 1 with lattice data from ref. [5], multiplied by $\chi_{\mathrm{q}} / T^{2}=0.897$. In the $\mathcal{O}\left(a_{s}^{4}\right)$ result, the renormalization scale has been varied within the range $\bar{\mu}=(1 \ldots 5) \times \bar{\mu}_{\text {ref }}$ (grey band). Right: The difference of the lattice and perturbative results, with $\chi_{\mathrm{q}}$ varied within its uncertainties. Errors are shown for $\chi_{\mathrm{q}} / T^{2}=0.897$ only.

these windows, the small remaining asymptotic value was subtracted from the correlator by hand, in order to allow for a Fourier transform to Minkowskian frequency space. We have now replaced the subtraction by another procedure; namely, the coefficient $a_{\ell_{\max }}$ for which the asymptotic value first crosses zero, is redefined to be

$$
a_{\ell_{\max }}^{\prime} \equiv-\sum_{\ell=0}^{\ell_{\max }-1} a_{\ell} .
$$

It turns out that this way the dependence on $\ell_{\max }$ is much milder than with the original procedure; in fact one does not even need to be close to a "window" but a plateau in $\sum_{\ell=0}^{\ell_{\max }} a_{\ell}^{2}$ would be sufficient as was envisaged in ref. [6]. The improvement is illustrated in fig. 3, obtained for the same data set (with the same simulated errors) as fig. 4 of ref. [7]; the results are substantially more stable, and equally close to the correct value ("input"), underestimating it by $\sim 25 \%$. (We have shown results for $N \equiv N_{\tau}=24$ to allow for a direct comparison with ref. [7]; the case $N=48$ corresponding to the resolution of ref. [5] shifts the results by $2-3 \%$ in the correct direction.)

Encouraged by this success, we have applied the algorithm to the difference $G_{i i}^{\text {diff }} \equiv G_{i i}^{\text {latt }}-G_{i i}^{\text {pert }}$ shown in fig. 2(right) (the corresponding spectral function is denoted by $\left.\rho_{i i}^{\text {diff }}\right) .{ }^{1}$ At very short distances no lattice data exists, so the difference has been kept fixed at its value at a chosen $\tau=\tau_{\min }$ (note that there is no reason for the difference to vanish). Various sources of systematic errors have

1 The data for $G_{i i}^{\text {diff }}$ as well as for a corresponding $G_{V}^{\text {diff }}$ can be obtained from the authors on request. The two differ by a constant mode; we stress that in the algorithm of ref. [6] or in the fit described in footnote 2 an exactly constant mode has no effect on the spectral function. been probed. We have checked that variations of the renormalization scale, as indicated in fig. 2(left), and variations of $T_{\mathrm{c}} / \Lambda_{\overline{\mathrm{MS}}}$ within a range $1.25 \pm 0.10$, consistent with e.g. ref. [25] (but also with earlier works, cf. sec. 4.2 of ref. [28]), have an effect smaller than variations of $\chi_{\mathrm{q}} / T^{2}=0.897(3)$, to which the continuum-extrapolated results were normalized in ref. [5]. The errors from the latter variation are shown in fig. 4(left). As can be anticipated from fig. 1, using 3 or 4-loop vacuum results would only lead to minor changes. Another source of systematic errors is the continuum extrapolation of ref. [5]; it might be prudent not to make use of results below $\tau T \simeq 0.20$ [5]. In fig. 4(right) we show the corresponding effects; in the following we restrict to $\tau_{\min } T=11 / 48$ which lies within a stable range.

It remains to consider statistical errors. The errors as shown in fig. 2 are strongly correlated, but only available for each $\tau T$ separately. We have then shifted the whole function upwards or downwards by the errors. It is important to stress once again that the algorithm of ref. [6] exactly projects out any constant contribution (i.e. Matsubara zero mode), so a uniform shift has no effect; the difference comes from the change in the shape. Results are shown in fig. 5; the variation from this rough implementation is smaller than systematic errors related to the analytic continuation.

\section{Conclusions}

Based on figs. 4, 5, and folding in an estimate of a downward systematic error as suggested by fig. 3 , we estimate

$$
\lim _{\omega \rightarrow 0^{+}} \frac{\rho_{i i}(\omega)}{\omega}=\lim _{\omega \rightarrow 0^{+}} \frac{\rho_{i i}^{\mathrm{diff}}(\omega)}{\omega} \gtrsim 0.3 T .
$$

Taking into account a factor 2 difference in the normalization of $\rho_{i i}$, the corresponding estimate was cited 

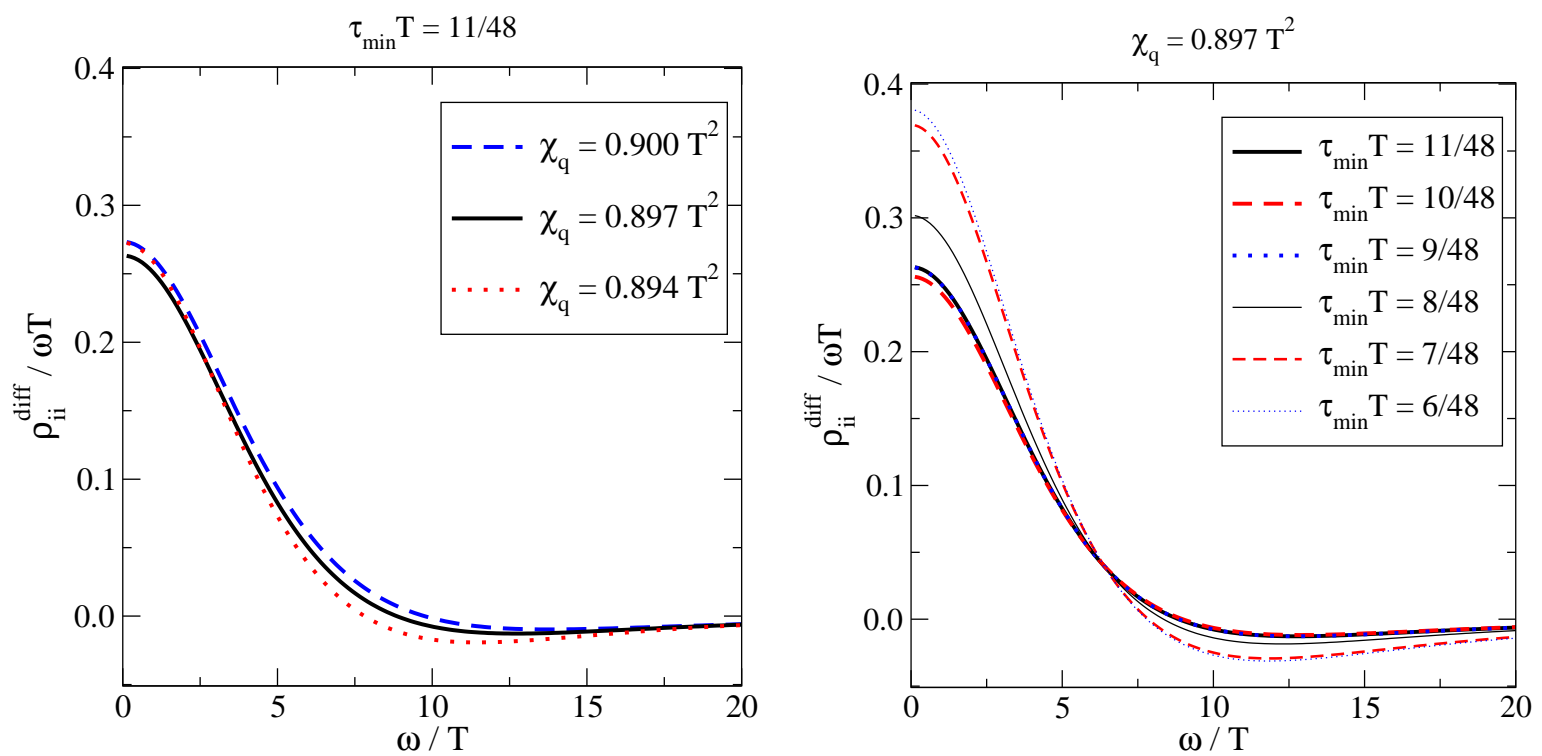

Fig. 4. Left: Dependence of the spectral function on $\chi_{\mathrm{q}} / T^{2}$, for a fixed $\tau_{\min } T=11 / 48$. Right: Dependence of the spectral function on $\tau_{\min } T$, for a fixed $\chi_{\mathrm{q}} / T^{2}=0.897$.

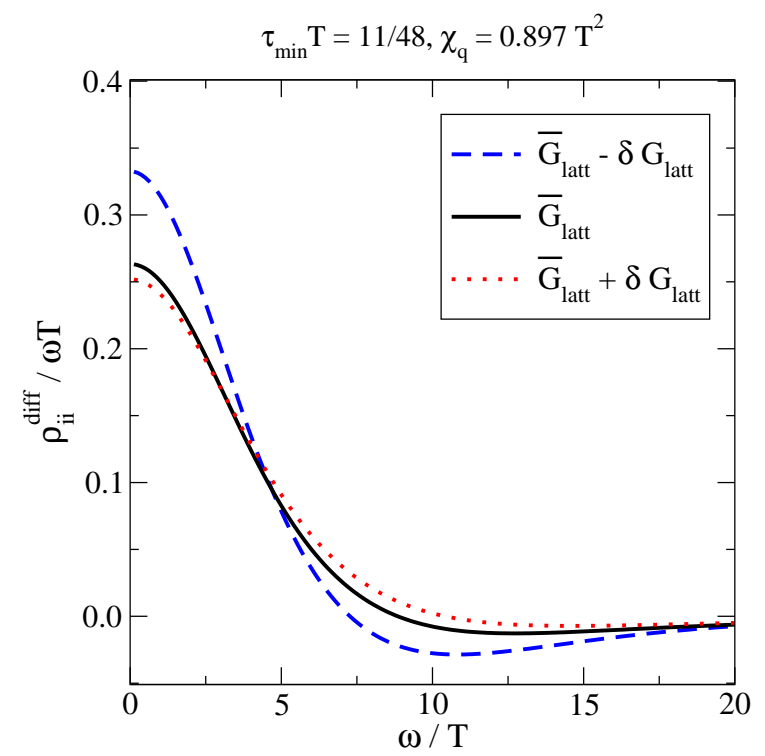

Fig. 5. Results obtained by modifying lattice data by adding or subtracting its statistical errors.

as $(1 \ldots 3) T$ in ref. [5], i.e. 3 - 9 times larger than our lower bound. (In ref. [29] only the lower limit was quoted, $D \chi_{\mathrm{q}} \simeq 0.33 T$. These estimates rely on eq. (2.3) in combination with non-logarithmic modellings of the UV part of $\rho_{i i} \cdot{ }^{2}$ ) From eq. (1.4), inserting $\chi_{\mathrm{q}}=0.897 T^{2}$, eq. (6.1)

\footnotetext{
${ }^{2}$ If the remainder from fig. 2(right) at $\tau T \geq 11 / 48$ is subjected to a 3-parameter fit to eq. (2.3) plus a $\tau$-independent constant, and errors are treated as uncorrelated, we obtain $D \chi_{\mathrm{q}} \simeq 0.30 T, \eta \simeq 1.1 T$, with $\chi^{2} /$ d.o.f. $\simeq 0.006$, numbers comparable with ref. [29]. The corresponding transport peak is markedly narrower than those in figs. 4, 5. Yet, as mentioned, the associated $G_{i i}^{\text {diff }}$ diverges at small $\tau$, whereas ours stays finite, as required by ref. [8]. If we cut off the large fre-
}

yields

$$
2 \pi T D \gtrsim 0.8
$$

The corresponding electrical conductivity from eq. (1.6) evaluates to $\sigma \gtrsim 0.07 e^{2} T$ for $N_{\mathrm{v}}=3, N_{\mathrm{f}}=0$.

These numbers are substantially smaller than those of the leading-order weak-coupling expansion [30,31], but they are intriguingly close to the AdS/CFT suggestion $2 \pi T D=1[32]$. However, the transport peak could be extremely narrow [33] and therefore our results should be interpreted as lower bounds, as has already been indicated by the notation. Continuum results are needed in the full $\tau$-range, and the resolution needs to be gradually increased, both in terms of statistical precision as well as in terms of $N_{\tau}$, in order to see whether the results stay put or show a slow evolution. (Unfortunately the functional dependences of the analytically continued results on statistical variance and $N_{\tau}$ are not easily extracted [6].)

In any case, the principal feasibility of carrying out a model-independent short-distance vacuum subtraction before attempting any analytic continuation or spectral modelling, thereby removing harmful UV contamination from the signal (cf. fig. 2), has hopefully become clear. This general philosophy can perhaps be applied to other correlators as well, for instance those related to components of the energy-momentum tensor, even if in that case temperature-dependent subtractions are needed in addition to the vacuum one [8].

quencies responsible for the divergence, for instance by defining $\rho_{i i}^{\left(L^{\prime}\right)}(\omega) \equiv \rho_{i i}^{(\mathrm{L})}(\omega) / \cosh \left(\frac{\omega}{2 \pi T}\right)$, then the fit result moves in our direction: $D \chi_{\mathrm{q}} \simeq 0.16 T, \eta \simeq 2.0 T$, with $\chi^{2} /$ d.o.f. $\simeq 0.005$. To resolve the correct physics it hence appears important to reach a good resolution for the continuum extrapolation of $G_{i i}^{\text {diff }}$ also at small $\tau$, verifying that it saturates to a constant value there. 


\section{Acknowledgements}

We thank H.-T. Ding for helpful discussions and providing us with lattice data from ref. [5], and O. Kaczmarek for useful discussions. Y.B. thanks the ITPP at Ecole Polytechnique Fédérale de Lausanne, were part of this work was carried out, for hospitality. M.L. acknowledges partial support by the BMBF under project 06BI9002.

\section{References}

1. J.I. Kapusta and C. Gale, Finite-Temperature Field Theory: Principles and Applications (Cambridge University Press, Cambridge, 2006).

2. H.B. Meyer, Transport Properties of the Quark-Gluon Plasma: A Lattice QCD Perspective, Eur. Phys. J. A 47 (2011) 86 [1104.3708].

3. S. Gupta, The electrical conductivity and soft photon emissivity of the QCD plasma, Phys. Lett. B 597 (2004) 57 [hep-lat/0301006].

4. G. Aarts, C. Allton, J. Foley, S. Hands and S. Kim, Spectral functions at small energies and the electrical conductivity in hot, quenched lattice QCD, Phys. Rev. Lett. 99 (2007) 022002 [hep-lat/0703008].

5. H.-T. Ding, A. Francis, O. Kaczmarek, F. Karsch, E. Laermann and $\mathrm{W}$. Soeldner, Thermal dilepton rate and electrical conductivity: An analysis of vector current correlation functions in quenched lattice QCD, Phys. Rev. D 83 (2011) 034504 [1012.4963].

6. G. Cuniberti, E. De Micheli and G.A. Viano, Reconstructing the thermal Green functions at real times from those at imaginary times, Commun. Math. Phys. 216 (2001) 59 [cond-mat/0109175].

7. Y. Burnier, M. Laine and L. Mether, A test on analytic continuation of thermal imaginary-time data, Eur. Phys. J. C 71 (2011) 1619 [1101.5534].

8. S. Caron-Huot, Asymptotics of thermal spectral functions, Phys. Rev. D 79 (2009) 125009 [0903.3958].

9. P.A. Baikov, K.G. Chetyrkin and J.H. Kühn, Hadronic Zand $\tau$-Decays in Order $\alpha_{s}^{4}$, Phys. Rev. Lett. 101 (2008) 012002 [0801.1821].

10. J. Casalderrey-Solana and D. Teaney, Heavy quark diffusion in strongly coupled $\mathcal{N}=4$ Yang-Mills, Phys. Rev. D 74 (2006) 085012 [hep-ph/0605199].

11. S. Caron-Huot, M. Laine and G.D. Moore, A Way to estimate the heavy quark thermalization rate from the lattice, JHEP 04 (2009) 053 [0901.1195].

12. A. Francis, O. Kaczmarek, M. Laine and J. Langelage, Towards a non-perturbative measurement of the heavy quark momentum diffusion coefficient, PoS LATTICE2011 (2011) 202 [1109.3941].

13. D. Banerjee, S. Datta, R. Gavai and P. Majumdar, Heavy Quark Momentum Diffusion Coefficient from Lattice QCD, Phys. Rev. D 85 (2012) 014510 [1109.5738].

14. F. Karsch, E. Laermann, P. Petreczky and S. Stickan, Infinite temperature limit of meson spectral functions calculated on the lattice, Phys. Rev. D 68 (2003) 014504 [heplat/0303017].

15. A. Vuorinen, Quark number susceptibilities of hot $Q C D$ up to $g^{6} \ln g$, Phys. Rev. D 67 (2003) 074032 [hep$\mathrm{ph} / 0212283]$.
16. Y. Burnier, M. Laine, J. Langelage and L. Mether, Colourelectric spectral function at next-to-leading order, JHEP 08 (2010) 094 [1006.0867].

17. M. Laine, A. Vuorinen and Y. Zhu, Next-to-leading order thermal spectral functions in the perturbative domain, JHEP 09 (2011) 084 [1108.1259].

18. R. Baier, B. Pire and D. Schiff, Dilepton production at finite temperature: Perturbative treatment at order $\alpha_{s}$, Phys. Rev. D 38 (1988) 2814.

19. Y. Gabellini, T. Grandou and D. Poizat, Electron-positron annihilation in thermal QCD, Annals Phys. 202 (1990) 436.

20. T. Altherr and P. Aurenche, Finite temperature $Q C D$ corrections to lepton-pair formation in a quark-gluon plasma, Z. Phys. C 45 (1989) 99.

21. G. Aarts and J.M. Martínez Resco, Transport coefficients, spectral functions and the lattice, JHEP 04 (2002) 053 [hepph/0203177].

22. P.A. Baikov, K.G. Chetyrkin and J.H. Kühn, $R(s)$ and hadronic $\tau$-Decays in Order $\alpha_{s}^{4}$ : technical aspects, Nucl. Phys. Proc. Suppl. 189 (2009) 49 [0906.2987].

23. T. van Ritbergen, J.A.M. Vermaseren and S.A. Larin, The four-loop $\beta$-function in quantum chromodynamics, Phys. Lett. B 400 (1997) 379 [hep-ph/9701390].

24. P.A. Baikov, K.G. Chetyrkin and J.H. Kühn, Adler Function, DIS sum rules and Crewther Relations, Nucl. Phys. Proc. Suppl. 205-206 (2010) 237 [1007.0478].

25. S. Borsanyi, G. Endrodi, Z. Fodor, S.D. Katz and K.K. Szabo, Lattice $S U(3)$ thermodynamics and the onset of perturbative behaviour, 1104.0013.

26. W. Florkowski and B.L. Friman, Spatial dependence of the finite temperature meson correlation function, Z. Phys. A 347 (1994) 271.

27. P.B. Arnold and L.G. Yaffe, Effective theories for real-time correlations in hot plasmas, Phys. Rev. D 57 (1998) 1178 [hep-ph/9709449].

28. M. Laine and Y. Schröder, Two-loop QCD gauge coupling at high temperatures, JHEP 03 (2005) 067 [hepph/0503061].

29. A. Francis and O. Kaczmarek, On the temperature dependence of the electrical conductivity in hot quenched lattice QCD, 1112.4802 .

30. P.B. Arnold, G.D. Moore and L.G. Yaffe, Transport coefficients in high temperature gauge theories. 1. Leading log results, JHEP 11 (2000) 001 [hep-ph/0010177].

31. P.B. Arnold, G.D. Moore and L.G. Yaffe, Transport coefficients in high temperature gauge theories. 2. Beyond leading log, JHEP 05 (2003) 051 [hep-ph/0302165].

32. P. Kovtun, D.T. Son and A.O. Starinets, Holography and hydrodynamics: Diffusion on stretched horizons, JHEP 10 (2003) 064 [hep-th/0309213].

33. G.D. Moore and J.-M. Robert, Dileptons, spectral weights, and conductivity in the quark-gluon plasma, hep$\mathrm{ph} / 0607172$. 